\title{
An Expression for Average Information Matrix for a Mixed Linear Multi-Component of Variance Model and REML Iteration Equations
}

\author{
Ichiro ASHIDA and Hiroaki IWAISAKI ${ }^{1}$ \\ Graduate School of Science and Technology, Niigata University, Niigata-shi 950-2181, Japan \\ ' Faculty of Agriculture, Niigata University, Niigata-shi 950-2181, Japan
}

(Received April 9, 1999 ; Accepted May 21, 1999)

\begin{abstract}
For the restricted maximum likelihood (REML) procedure, which is an iterative method and is in common use to estimate variance components in animal breeding researches and applications, there have been proposed the computing algorithms of several kinds. Focussing on the so-called average information (AI) algorithm and assuming a mixed linear, multi-component of variance model, in this study, an expression for the AI matrix in which the vector of predicted residuals is not contained is derived. The resulting elements of the AI matrix are expressed in terms of quadratic and bilinear forms for the vectors of observations and the mixed model solutions. Replacing the Hessian matrix by the current AI matrix, a quasi-Newton type procedure is defined for REML estimation. Although a generalized inverse of the mixed model coefficient matrix and direct solutions to the mixed model equations are required to form the current AI matrix, use of sparse matrix tools makes the proposed numerical technique efficient. As an illustration, some computing performance of the current REML procedure is shown, analyzing a data set on carcass weight in the Japanese Black cattle.
\end{abstract}

Animal Science Journal 70 (5) : 282-289, 1999

Key words : Restricted maximum likelihood, Multiple components of variance, Average information, Quadratic and bilinear forms, Numerical technique

For the restricted maximum likelihood (REML) estimation of variance components in mixed linear models $^{(1)}$ and thereby genetic parameters, the socalled average information (AI) algorithm ${ }^{9}$ is recently attracting the interest of animal breeders because of its computational advantage over the expectationmaximization (EM) $)^{3,5)}$ and the derivative-free ${ }^{4,15)}$ algorithms. The AI algorithm utilizes the average of the observed and expected information in place of the expected information as used in the Fisher scoring method. As a consequence, with this algorithm, the calculation of traces of matrix products of large order can be avoided. For instance, a numerical technique using the AI algorithm for REML estimation is based on an expression for the AI matrix whose elements are defined as vector products including the vector of predicted residuals ${ }^{9)}$.

For the AI matrix, Ashida and Iwaisaki ${ }^{1)}$ described an alternative expression in terms of the vectors of observations and the mixed model solutions in which the vector of predicted residuals is not included. The authors also proposed a numerical technique for REML estimation using the alternative expression. The alternative expression would be useful in understanding the difference between AI and the expected information from the viewpoint of the relations to the mixed model equations and solutions. Although the numerical technique straightforwardly using the alternative expression definitely requires the calculation of a generalized inverse of the mixed model coefficient matrix, it appears that its use with sparse matrix tools is one practical approach even for a relatively large 
data set. However, the expression given by Ashida and Iwaisaki ${ }^{1)}$ was just for the simple model that contains only one random effect, or animal effect, other than the residual effect.

Thus, in this paper, we derive the corresponding expression for the AI matrix in the case of a mixed linear, multi-component of variance model and describe REML iteration equations of the quasi-Newton type using the AI matrix derived.

\section{Theoretical Consideration}

\section{The model and mixed model solutions}

We assume a mixed linear model, as follows

$$
\mathbf{y}=\mathbf{X b}+\mathbf{Z} \mathbf{u}+\mathbf{e},
$$

where $\mathbf{y}$ is an $N \times 1$ vector of observations, $\mathbf{b}$ is a $p \times$ 1 vector of fixed effects, $\mathbf{u}$ is a $q \times 1$ vector of random effects, $\mathrm{e}$ is an $N \times 1$ vector of random residuals, $\mathrm{X}$ is the $N \times p$ incidence matrix for fixed effects, and $\mathbf{Z}$ is the $N \times q$ incidence matrix for random effects, with $\mathbf{u}$ and $\mathbf{Z}$ being partitioned as<smiles>[Y][Z20][Y]</smiles>

respectively, where $s$ is the number of random factors except for the residual term, and $\sum_{i=1}^{s} q_{i}=q$ with representing the order of $\mathbf{u}_{i}$ by $q_{i}$.

It is assumed that

$$
\mathbf{y} \sim \mathbf{N}(\mathbf{X b}, \mathbf{V})
$$

$$
\mathrm{E}\left[\begin{array}{c}
\mathbf{u}_{1} \\
\mathbf{u}_{2} \\
\vdots \\
\mathbf{u}_{i} \\
\vdots \\
\mathbf{u}_{s} \\
\mathbf{e}
\end{array}\right]=\left[\begin{array}{c}
\mathbf{0}_{\left(q_{1} \times 1\right)} \\
\mathbf{0}_{\left(q_{2} \times 1\right)} \\
\vdots \\
\mathbf{0}_{\left(q_{i} \times 1\right)} \\
\vdots \\
\mathbf{0}_{\left(q_{s} \times 1\right)} \\
\mathbf{0}_{(N \times 1)}
\end{array}\right]
$$

$$
\operatorname{and} \operatorname{Var}\left[\begin{array}{c}
\mathbf{u}_{1} \\
\mathbf{u}_{2} \\
\vdots \\
\mathbf{u}_{i} \\
\vdots \\
\mathbf{u}_{s} \\
\mathbf{e}
\end{array}\right]=\left[\begin{array}{ccccccc}
\mathbf{G}_{1} \sigma_{1}^{2} & \mathbf{0}_{\left(q_{1} \times q_{2}\right)} & \cdots & \mathbf{0}_{\left(q_{1} \times q_{i}\right)} & \cdots & \mathbf{0}_{\left(q_{1} \times q_{s}\right)} & \mathbf{0}_{\left(q_{1} \times N\right)} \\
& \mathbf{G}_{2} \sigma_{2}^{2} & \cdots & \mathbf{0}_{\left(q_{2} \times q_{i}\right)} & \cdots & \mathbf{0}_{\left(q_{2} \times q_{s}\right)} & \mathbf{0}_{\left(q_{2} \times N\right)} \\
& & \ddots & \vdots & & \vdots & \vdots \\
& & & \mathbf{G}_{i} \sigma_{i}^{2} & \cdots & \mathbf{0}_{\left(q_{i} \times q_{s}\right)} & \mathbf{0}_{\left(q_{i} \times N\right)} \\
& & & & \ddots & \vdots & \vdots \\
& S y m . & & & & \mathbf{G}_{s} \sigma_{s}^{2} & \mathbf{0}_{\left(q_{s} \times N\right)} \\
& & & & & & \mathbf{I}_{(N \times N)} \sigma_{0}^{2}
\end{array}\right] \text {, }
$$

supposing that there are no covariances between the random vectors. Consequently, we suppose that

$$
\operatorname{Var}[\mathbf{y}]=\mathbf{V}=\sum_{i=1}^{s} \mathbf{Z}_{i} \mathbf{G}_{i} \mathbf{Z}_{i}^{\prime} \sigma_{i}^{2}+\mathbf{I} \sigma_{0}^{2} .
$$

Then the mixed model equations of Henderson ${ }^{6-8)}$ are of the following form

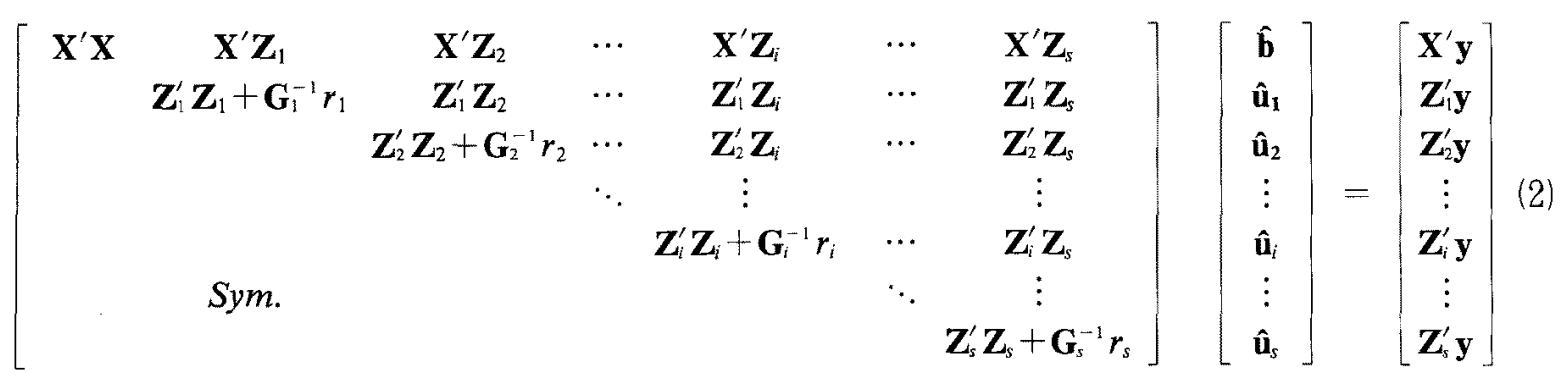


with $r_{i}=\sigma_{0}^{2} \sigma_{i}^{-2}$. Denotying a generalized inverse of the coefficient matrix of equation (2) by

$$
\mathbf{C}=\left[\begin{array}{ccccccc}
\mathbf{C}_{b b} & \mathbf{C}_{b 1} & \mathbf{C}_{b 2} & \cdots & \mathbf{C}_{b i} & \cdots & \mathbf{C}_{b s} \\
& \mathbf{C}_{11} & \mathbf{C}_{12} & \cdots & \mathbf{C}_{1 i} & \cdots & \mathbf{C}_{1 s} \\
& & \mathbf{C}_{22} & \cdots & \mathbf{C}_{2 i} & \cdots & \mathbf{C}_{2 s} \\
& & & \ddots & \vdots & & \vdots \\
& & & & \mathbf{C}_{i i} & \cdots & \mathbf{C}_{i s} \\
& S y m . & & & & \ddots & \vdots \\
& & & & & & \mathbf{C}_{s s}
\end{array}\right],
$$

the mixed model solutions are written as

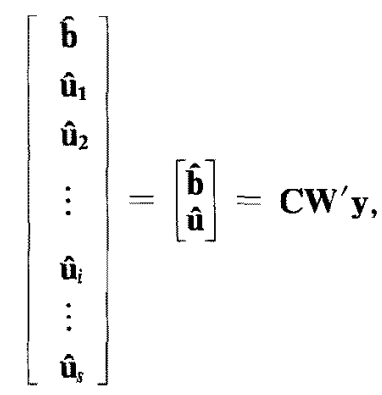

where

$$
\mathbf{W}=\left[\begin{array}{ll}
\mathbf{X} & \mathbf{Z}
\end{array}\right] .
$$

\section{The REML log-likelihood and the derivatives}

The REML likelihood is the likelihood of linear functions of the observation vector with expectations zero, or error contrasts. Following Harville's ${ }^{5)}$ and Searle's ${ }^{13)}$ work, the logarithm of the REML likelihood is written as

$$
\begin{aligned}
L= & -\frac{1}{2}\left\{\text { const. }+\log |\mathbf{V}|+\log \left|\mathbf{X}^{*^{\prime}} \mathbf{V}^{-1} \mathbf{X}^{*}\right|\right. \\
& \left.+\mathbf{y}^{\prime} \mathbf{P y}\right\}
\end{aligned}
$$

with

$$
\mathbf{y}^{\prime} \mathbf{P y}=(\mathbf{y}-\mathbf{X} \hat{\mathbf{b}})^{\prime} \mathbf{V}^{-1}(\mathbf{y}-\mathbf{X} \hat{\mathbf{b}}),
$$

where $\mathbf{X}^{*}$ is a full rank submatrix of $\mathbf{X}$, and

$$
\begin{aligned}
\mathbf{P} & =\mathbf{V}^{-1}-\mathbf{V}^{-1} \mathbf{X}^{*}\left(\mathbf{X}^{*} \mathbf{V}^{-1} \mathbf{X}^{*}\right)^{-1} \mathbf{X}^{* \prime} \mathbf{V}^{-1} \\
& =\mathbf{V}^{-1}-\mathbf{V}^{-1} \mathbf{X}\left(\mathbf{X}^{\prime} \mathbf{V}^{-1} \mathbf{X}\right)^{-} \mathbf{X}^{\prime} \mathbf{V}^{-1}
\end{aligned}
$$

Then, denoting $\partial \mathbf{V} / \partial \sigma_{i}^{2}$ by $\mathbf{V}_{i}$, differentiation of equation (4) with respect to the $\sigma_{i}^{2}$ 's $(i=0,1, \cdots, s)$ gives

$$
\frac{\partial L}{\partial \sigma_{i}^{2}}=-\frac{1}{2}\left\{\operatorname{tr}\left(\mathbf{P} \mathbf{V}_{i}\right)-\mathbf{y}^{\prime} \mathbf{P} \mathbf{V}_{i} \mathbf{P y}\right\}
$$

Furthermore, the second-order partial derivatives of $L$ are yielded as

$$
\frac{\partial^{2} L}{\partial \sigma_{l}^{2} \partial \sigma_{j}^{2}}=\frac{1}{2} \operatorname{tr}\left(\mathbf{P} \mathbf{V}_{i} \mathbf{P} \mathbf{V}_{j}\right)-\mathbf{y}^{\prime} \mathbf{P} \mathbf{V}_{i} \mathbf{P} \mathbf{V}_{j} \mathbf{P y}
$$

with $\partial^{2} \mathbf{V} / \partial \sigma_{i}^{2} \partial \sigma_{j}^{2}=0$, because for the model (1), $\mathbf{V}$ is linear in the parameters, i.e., $\sigma_{i}^{2}(i=0,1, \cdots, s)$. The expectation of equation (6) is given by

$$
\mathbf{E}\left[\frac{\partial^{2} L}{\partial \sigma_{i}^{2} \partial \sigma_{j}^{2}}\right]=-\frac{1}{2} \operatorname{tr}\left(\mathbf{P} \mathbf{V}_{i} \mathbf{P} \mathbf{V}_{j}\right)
$$

since $\mathbf{P X}=\mathbf{0}$ and $\mathbf{P V P}=\mathbf{P}$.

The average information and the Johnson and Thompson expression

For equations (6) and (7), taking an average leads to

$$
\begin{aligned}
& \frac{1}{2}\left(-\frac{\partial^{2} L}{\partial \sigma_{i}^{2} \partial \sigma_{j}^{2}}+\mathrm{E}\left[-\frac{\partial^{2} L}{\partial \sigma_{i}^{2} \partial \sigma_{j}^{2}}\right]\right) \\
& \quad=\frac{1}{2} \mathbf{y}^{\prime} \mathbf{P} \mathbf{V}_{i} \mathbf{P} \mathbf{V}_{j} \mathbf{P y},
\end{aligned}
$$

which is called the average information ${ }^{9)}$, or AI.

Since $\mathbf{V}_{i}=\mathbf{Z}_{i} \mathbf{G}_{i} \mathbf{Z}_{i}^{\prime}(i=1,2, \cdots, s)$ and $\mathbf{V}_{0}=\mathbf{I}$, we have the following vectors

$$
\begin{aligned}
\boldsymbol{\alpha}_{i} & =\mathbf{V}_{i} \mathbf{P y} \\
& =\mathbf{Z}_{i} \hat{\mathbf{u}}_{i} \sigma_{i}^{-2}
\end{aligned}
$$

and

$$
\begin{aligned}
\boldsymbol{\alpha}_{0} & =\mathbf{P y} \\
& =\hat{\mathbf{e}} \sigma_{0}^{-2},
\end{aligned}
$$

as shown by Johnson and Thompson"), where ê is the vector of residuals predicted for the model (1). Furthermore, since $\mathbf{P}$ is the matrix transforming observations into residuals, as one strategy for computing, $\beta_{i}$ ( $=\mathbf{P} \alpha_{j}$ ) can be calculated as the residuals from solving the mixed model equations for the model (1) with y replaced by $\alpha_{j}$.

As presented by Johnson and Thompson ${ }^{9)}$, hence, the average information $\left(a_{i j}\right)$ is given as 
An Expression for Average Information Matrix

$$
a_{i j}=\frac{1}{2} \alpha_{i}^{\prime} \beta_{j}
$$

\section{Alternative expression for the AI matrix}

For simplicity, we will first assume that $s=2$, namely

$$
\mathbf{y}=\mathbf{X b}+\mathbf{Z}_{1} \mathbf{u}_{1}+\mathbf{Z}_{2} \mathbf{u}_{2}+\mathbf{e}
$$

and will give an alternative expression for the AI matrix, and then we will yield the general expression pertaining to the model (1).

For the model (12), the element $a_{00}$ of the AI matrix, from equation (8), is written as

$$
\frac{1}{2} \mathbf{y}^{\prime} \mathbf{P}^{3} \mathbf{y}=\frac{1}{2} \mathbf{y}^{\prime}\left(I-\mathbf{W C W}^{\prime}\right)^{3} \mathbf{y} \sigma_{0}^{-6}
$$

Now, define a matrix $\mathbf{B}$ as

$$
\mathbf{B}=\left[\begin{array}{ccc}
\mathbf{0} & \mathbf{0} & \mathbf{0} \\
\mathbf{0} & \mathbf{G}_{1}^{-1} r_{1} & \mathbf{0} \\
\mathbf{0} & \mathbf{0} & \mathbf{G}_{2}^{-1} r_{2}
\end{array}\right]
$$

Then we have

$$
\begin{aligned}
\mathbf{C W}^{\prime} \mathbf{W} & =\mathbf{I}-\mathbf{C B} \\
& =\left[\begin{array}{ccc}
\mathbf{I} & -\mathbf{C}_{01} \mathbf{G}_{1}^{-1} \boldsymbol{r}_{1} & -\mathbf{C}_{02} \mathbf{G}_{2}^{-1} \boldsymbol{r}_{2} \\
\mathbf{0} & \mathbf{I}-\mathbf{C}_{11} \mathbf{G}_{1}^{-1} \boldsymbol{r}_{1} & -\mathbf{C}_{12} \mathbf{G}_{2}^{-1} \boldsymbol{r}_{2} \\
\mathbf{0} & -\mathbf{C}_{12}^{\prime} \mathbf{G}_{1}^{-1} \boldsymbol{r}_{1} & \mathbf{I}-\mathbf{C}_{22} \mathbf{G}_{2}^{-1} \boldsymbol{r}_{2}
\end{array}\right] \\
& =\left[\begin{array}{lll}
\mathbf{H}_{00} & \mathbf{H}_{10} & \mathbf{H}_{20} \\
\mathbf{H}_{01} & \mathbf{H}_{11} & \mathbf{H}_{21} \\
\mathbf{H}_{02} & \mathbf{H}_{12} & \mathbf{H}_{22}
\end{array}\right] \\
& \equiv\left[\begin{array}{llll}
\mathbf{H}_{0} & \vdots \mathbf{H}_{1} & \mathbf{H}_{2}
\end{array}\right] .
\end{aligned}
$$

So, equation (13) is further written as

$$
\frac{1}{2} \mathbf{y}^{\prime}\left(\mathbf{I}-\mathbf{W C W}-\mathbf{W C B C W}-\mathbf{W C B C B C W ^ { \prime }}\right) \mathbf{y} \sigma_{0}^{-.6}
$$

Then, using equation (3) and noting that

$$
\mathbf{y}^{\prime} \mathbf{W C B}=\left[\mathbf{0} \vdots \hat{\mathbf{u}}_{1}^{\prime} \mathbf{G}_{1}^{-1} r_{1} \vdots \hat{\mathbf{u}}_{2}^{\prime} \mathbf{G}_{2}^{-1} r_{2}\right],
$$

with some arrangement we finally find

$$
\begin{aligned}
a_{00}= & \frac{1}{2}\left(\mathbf{y}^{\prime} \mathbf{y}-\hat{\mathbf{b}}^{\prime} \mathbf{X}^{\prime} \mathbf{y}-\hat{\mathbf{u}}_{1}^{\prime} \mathbf{Z}_{1}^{\prime} \mathbf{y}-\hat{\mathbf{u}}_{2}^{\prime} \mathbf{Z}_{2}^{\prime} \mathbf{y}\right. \\
& -\hat{\mathbf{u}}_{1}^{\prime} \mathbf{G}_{1}^{-1} \hat{\mathbf{u}}_{1} r_{1}-\hat{\mathbf{u}}_{2}^{\prime} \mathbf{G}_{2}^{-1} \hat{\mathbf{u}}_{2} r_{2} \\
& -\hat{\mathbf{u}}_{1}^{\prime} \mathbf{G}_{1}^{-1} \mathbf{C}_{11} \mathbf{G}_{1}^{-1} \hat{\mathbf{u}}_{1} r_{1}^{2}-\hat{\mathbf{u}}_{2}^{\prime} \mathbf{G}_{2}^{-1} \mathbf{C}_{22} \mathbf{G}_{2}^{-1} \hat{\mathbf{u}}_{2} r_{2}^{2}
\end{aligned}
$$

$$
\left.-2 \hat{\mathbf{u}}_{1}^{\prime} \mathbf{G}_{1}^{-1} \mathbf{C}_{12} \mathbf{G}_{2}^{-1} \hat{\mathbf{u}}_{2} r_{1} r_{2}\right) \sigma_{0}^{-6}
$$

Next, $a_{0 i}$ for $i=1,2$ are expressed as

$$
\frac{1}{2} \mathbf{y}^{\prime} \mathbf{P}^{2} \mathbf{V}_{i} \mathbf{P y}=\frac{1}{2} \mathbf{y}^{\prime}\left(\mathbf{I}-\mathbf{W C W} \mathbf{W}^{\prime}\right)^{2} \mathbf{Z}_{i} \hat{\mathbf{u}}_{i} \sigma_{i}^{-2} \sigma_{0}^{-4},
$$

which are given as

$$
\frac{1}{2}\left(\mathbf{y}^{\prime} \mathbf{Z}_{i} \hat{\mathbf{u}}_{i}-\mathbf{y}^{\prime} \mathbf{W} \mathbf{H}_{i} \hat{\mathbf{u}}_{i}-\mathbf{y}^{\prime} \mathbf{W} \mathbf{C B H} \hat{H}_{i} \hat{\mathbf{u}}_{i}\right) \sigma_{i}^{-2} \sigma_{0}^{-4}
$$

Hence, we obtain

$$
\begin{aligned}
a_{0 i}= & \frac{1}{2}\left(\hat{\mathbf{u}}_{i}^{\prime} \mathbf{G}_{i}^{-1} \mathbf{C}_{i i} \mathbf{G}_{i}^{-1} \hat{\mathbf{u}}_{i} r_{i}^{2}\right. \\
& \left.+\hat{\mathbf{u}}_{1}^{\prime} \mathbf{G}_{1}^{-1} \mathbf{C}_{12} \mathbf{G}_{2}^{-1} \hat{\mathbf{u}}_{2} \boldsymbol{r}_{1} r_{2}\right) \sigma_{i}^{-2} \sigma_{0}^{-4}
\end{aligned}
$$

Similarly, $a_{i i}$ for $i=1,2$ are written as

$$
\begin{aligned}
\frac{1}{2} \mathbf{y}^{\prime} \mathbf{P} \mathbf{V}_{i} \mathbf{P} \mathbf{V}_{i} \mathbf{P y}= & \frac{1}{2} \hat{\mathbf{u}}_{i}^{\prime} \mathbf{Z}_{i}^{\prime}\left(\mathbf{I}-\mathbf{W C W} \mathbf{W}^{\prime}\right) \\
& \mathbf{Z}_{i} \hat{\mathbf{u}}_{i} \sigma_{i}^{-4} \sigma_{0}^{-2}
\end{aligned}
$$

These equations can be arranged as

$$
\frac{1}{2} \hat{\mathbf{u}}_{i}^{\prime} \mathbf{Z}_{i}^{\prime} \mathbf{W} \mathbf{C}_{i}^{\prime} \mathbf{G}_{i}^{-1} \hat{\mathbf{u}}_{i} r_{i} \sigma_{j}^{-4} \sigma_{0}^{-2} \text {. }
$$

Thus, further using $\mathbf{Z}_{j}^{\prime} \mathbf{W} \mathbf{C}_{j}^{\prime}=\mathbf{H}_{i l}$ leads to

$$
a_{i i}=\frac{1}{2}\left(\hat{\mathbf{u}}_{i}^{\prime} \mathbf{G}_{i}^{-1} \hat{\mathbf{u}}_{i} r_{i}-\hat{\mathbf{u}}_{i}^{\prime} \mathbf{G}_{i}^{-1} \mathbf{C}_{i i} \mathbf{G}_{i}^{-1} \hat{\mathbf{u}}_{i} r_{i}^{2}\right) \sigma_{i}^{-4} \sigma_{0}^{-2}
$$

Finally, for the element $a_{12}$, we have the following bilinear form

$$
\begin{aligned}
\frac{1}{2} \mathbf{y}^{\prime} \mathbf{P V} \mathbf{V}_{1} \mathbf{P V} \mathbf{P y}= & \frac{1}{2} \hat{\mathbf{u}}_{1}^{\prime} \mathbf{Z}_{1}^{\prime}\left(\mathbf{I}-\mathbf{W C W} \mathbf{W}^{\prime}\right) \\
& \mathbf{Z}_{2} \hat{\mathbf{u}}_{2} \sigma_{2}^{-2} \sigma_{1}^{-2} \sigma_{0}^{-2}
\end{aligned}
$$

This becomes

$$
\frac{1}{2}\left(\hat{\mathbf{u}}_{1}^{\prime} \mathbf{Z}_{1}^{\prime} \mathbf{Z}_{2} \hat{\mathbf{u}}_{2}-\hat{\mathbf{u}}_{1}^{\prime} \mathbf{Z}_{1} \mathbf{W} \mathbf{H}_{2} \hat{\mathbf{u}}_{2}\right) \sigma_{2}^{-2} \sigma_{1}^{-2} \sigma_{0}^{-2},
$$

which reduces to

$$
\frac{1}{2} \hat{\mathbf{u}}_{1}^{\prime} \mathbf{Z}_{1}^{\prime} \mathbf{W} \mathbf{C}_{2}^{\prime} \mathbf{G}_{2}^{-1} \hat{\mathbf{u}}_{2} \boldsymbol{r}_{2} \sigma_{2}^{-2} \sigma_{1}^{-2} \sigma_{0}^{-2}
$$

Noting that $\mathbf{Z}_{1}^{\prime} \mathbf{W} \mathbf{C}_{2}^{\prime}=-\mathbf{G}_{1}^{-1} \mathbf{C}_{12} r_{1}$, we therefore get

$$
a_{12}=-\frac{1}{2} \hat{\mathbf{u}}_{1}^{\prime} \mathbf{G}_{1}^{-1} \mathbf{C}_{12} \mathbf{G}_{2}^{-1} \hat{\mathbf{u}}_{2} r_{1} r_{2} \sigma_{2}^{-2} \sigma_{1}^{-2} \sigma_{0}^{-2}
$$




\section{ASHIDA and IWAISAKI}

Table 1. Computing performance of the current procedure

\begin{tabular}{|c|c|c|c|c|c|c|c|}
\hline \multirow{2}{*}{$\begin{array}{c}\text { Convergence } \\
\text { criterion }\end{array}$} & \multirow{2}{*}{ Procedure } & \multicolumn{3}{|c|}{ Estimate $\left(\mathrm{kg}^{2}\right)$} & \multirow{2}{*}{$\begin{array}{c}\text { Number of } \\
\text { iterations }\end{array}$} & \multicolumn{2}{|c|}{ Computing time } \\
\hline & & $\sigma_{2}^{2}$ & $\sigma_{1}^{2}$ & $\sigma_{0}^{2}$ & & Absolute (s) & Relative $^{\perp)}$ \\
\hline \multirow[t]{2}{*}{$10^{-6}$} & AIREML & 200.34 & $1,205.75$ & 593.63 & 9 & 38 & 1 \\
\hline & EMREML & 200.34 & $1,205.31$ & 593.94 & 1,098 & 1,876 & 49.4 \\
\hline \multirow[t]{2}{*}{$10^{-9}$} & AIREML & 200.34 & $1,205.75$ & 593.63 & 13 & 50 & 1 \\
\hline & EMREML & 200.34 & $1,205.75$ & 593.63 & 2,350 & 3,999 & 80.0 \\
\hline
\end{tabular}

${ }^{1)}$ Relative to AIREML under a given criterion for convergence.

Using the similar approach, it is then straightforward to derive the general form of the alternative expression. Letting $\theta$ denote the vector of the para- meters to be estimated, i.e.,

$$
\boldsymbol{\theta}^{\prime}=\left[\begin{array}{lllll}
\sigma_{0}^{2} & \sigma_{1}^{2} & \sigma_{2}^{2} & \cdots & \sigma_{s}^{2}
\end{array}\right],
$$

it may be written as

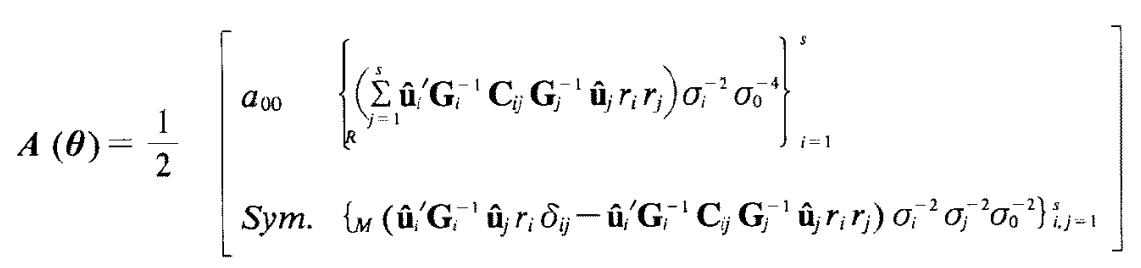

with

$$
\boldsymbol{a}_{00}=\left(\mathbf{y}^{\prime} \mathbf{y}-\hat{\mathbf{b}}^{\prime} \mathbf{X}^{\prime} \mathbf{y}-\sum_{i=1}^{s} \hat{\mathbf{u}}_{i}^{\prime} \mathbf{Z}_{i}^{\prime} \mathbf{y}-\sum_{i=1}^{s} \hat{\mathbf{u}}_{i}^{\prime} \mathbf{G}_{i}^{-1} \hat{\mathbf{u}}_{i} r_{i}-\sum_{i=1}^{s} \sum_{j=1}^{s} \hat{\mathbf{u}}_{i}^{\prime} \mathbf{G}_{i}^{-1} \mathbf{C}_{i j} \mathbf{G}_{j}^{-1} \hat{\mathbf{u}}_{j} r_{i} \boldsymbol{r}_{j}\right) \sigma_{0}^{-6},
$$

where the notation $\left\{_{R} \cdot\right\}_{i=1}^{s}$ represents a row vector with the $s$ elements for $i=1$ to $s$ inside the brace, $\left\{_{w} \cdot\right\}_{i, j=1}^{s}$ stands for a matrix of order $s \times s$ whose elements for $i=1$ to $s$ and $j=1$ to $s$ are given in the brace, and $\delta_{i j}$ is the Kronecker delta with $\delta_{i i}=1$ and $\delta_{i j}=0$ for $i \neq j$.

\section{A Newton type procedure for REML estimation}

The derived expression for the AI matrix, or equation (21), can be used for REML estimation of the variance components. Replacing the Hessian matrix by $A(\theta)$, a set of equations for the iterative estimation can be written as

$$
\hat{\boldsymbol{\theta}}_{t+1}=\hat{\boldsymbol{\theta}}_{t}+\left[\boldsymbol{A}\left(\hat{\boldsymbol{\theta}}_{t}\right)\right]^{-1} \partial L / \partial \boldsymbol{\theta} \mid \boldsymbol{\theta}=\hat{\boldsymbol{\theta}}_{t}
$$

where $\hat{\theta}_{t+1}$ and $\hat{\theta}_{t}$ are the estimates of $\theta$ in the $t+1^{\text {th }}$ and the $t^{\text {th }}$ iterations, $\boldsymbol{A}\left(\hat{\theta}_{t}\right)$ is the matrix $\boldsymbol{A}(\boldsymbol{\theta})$ calculated at $\boldsymbol{\theta}=\hat{\boldsymbol{\theta}}_{t}$, and $\partial L / \partial \boldsymbol{\theta} \mid \boldsymbol{\theta}=\hat{\boldsymbol{\theta}}_{t}$ is the gradient vector, or the vector of first derivatives of the REML $\log$-likelihood function with $\theta$ replaced by $\hat{\boldsymbol{\theta}}_{t}$.

\section{Numerical Analysis}

We will briefly demonstrate the computing performance of the estimation procedure based on equation (22) (denoted as AIREML), compared to the EM algorithm (EMREML).

The data used were 4,412 records on carcass weight in the Japanese Black fattening cattle and were the same data as utilized by Ashida and Iwaisaki ${ }^{1)}$. A total of 12,204 animals, including 7,792 animals in the pedigree file, were analyzed. The operational animal model fitted was the same as used by them except that farm effect was treated as random here. Farm, animal and residual variances were denoted by $\sigma_{2}^{2}, \sigma_{1}^{2}$ and $\sigma_{0}^{2}$, respectively. For details on the fixed effects contained in the model, the initial values, the computer facilities used and so on, see Ashida and Iwaisak $i^{1}$. For $\sigma_{2}^{2}$, the pre-assigned value was determined, 
An Expression for Average Information Matrix

assuming that the proportion of the variance to the total variance is $20 \%$. Two levels of the convergence criterion were considered : changes of the variance ratios between two consecutive rounds were all less than $10^{-6}$ or $10^{-9}$.

The current AIREML procedure performed very well in the analysis. The results are shown in Table 1. The EMREML procedure necessitated more than one thousand and two thousand rounds of iteration to attain convergence for the criteria of $10^{-6}$ and $10^{-9}$, respectively, but AIREML required only nine and thirteen rounds, respectively. Moreover, it should be noted that the AIREML estimates agreed within decimal places given in the table for the two convergence criteria, while the corresponding estimates by EMREML did not agree for $\sigma_{1}^{2}$ and $\sigma_{0}^{2}$. With the criterion of $10^{-6}$, the computing time in EMREML was about fifty times longer than that in AIREML. When the criterion was severer, the difference between the computing time for the two procedures became larger, as expected.

\section{Discussion}

As has been seen from the interrelations of equations (6), (7) and (8), apart from a constant, the AI equals the second partial derivatives of the quadratic form $\mathbf{y}^{\prime} \mathbf{P y}$ with respect to the variance components, which corresponds to the data part of the observed information. For the AI matrix whose elements are the AI, an expression has been given herein assuming a mixed linear multi-component of variance model. In the current elements of the AI matrix, no vector of the predicted residuals $\hat{\mathbf{e}}$ is contained, and the mixed model solutions and a generalized inverse of the mixed model coefficient matrix are rather included. That is, the quadratic and bilinear forms of particular types, or $\hat{\mathbf{u}}_{j}^{\prime} \mathbf{G}_{i}^{-1} \mathbf{C}_{i i} \mathbf{G}_{i}^{-1} \hat{\mathbf{u}}_{i}$ and $\hat{\mathbf{u}}_{i}^{\prime} \mathbf{G}_{i}^{-1} \mathbf{C}_{i j} \mathbf{G}_{j}^{-1} \hat{\mathbf{u}}_{j}(i \neq j)$ are involved in certain elcments of the current AI matrix. It is seen that these bilinear forms are the terms specific to the multi-component of variance model.

For the EM type algorithm for REML estimation, which is an algorithm using the first partial derivatives of the REML log-likelihood with respect to the parameters, iteration equations of one type are of the form including the quadratic form of $\hat{\mathbf{e}}$, or $\hat{\mathbf{e}}^{\prime} \hat{\mathbf{e}}$, while those of another type do not contain any quadratic forms of $\hat{\mathbf{e}}^{5)}$. The latter equations involve the quadratic form of the following type

$$
Q=\mathbf{y}^{\prime} \mathbf{y}-\hat{\mathbf{b}}^{\prime} \mathbf{X}^{\prime} \mathbf{y}-\sum_{i=1}^{s} \hat{\mathbf{u}}_{i}^{\prime} \mathbf{Z}_{i}^{\prime} \mathbf{y}-\sum_{i=1}^{s} \hat{\mathbf{u}}_{i}^{\prime} \mathbf{G}_{i}{ }^{\prime} \hat{\mathbf{u}}_{i} r_{i},
$$

of which the basic form was derived by Thompson ${ }^{16)}$. In this context, apart from a constant and the residual variance, one element of the AI matrix originally includes the quadratic form $\hat{\mathbf{e}}^{\prime} \mathbf{P} \hat{\mathbf{e}}$ and consequently $\hat{\mathbf{e}} \mathbf{\hat { \mathbf { e } }}$. Instead of $\hat{\mathbf{e}}$, our alternative expression utilizes the quadratic form of $\mathbf{y}$ including $Q$ and therefore is likely to be an application of the Thompson approach mentioned above to the second partial derivatives of $\mathbf{y}^{\prime} \mathbf{P y}$ with respect to the parameters.

Of mixed linear models with multiple components of variance, the simpler model (12) corresponds to a class of animal models often used in animal breeding applications. Some examples may be a repeatability model for repeated records on each animal and a litter effect model to take account of the common environmental variance for full siblings. Moreover, a model with additive genetic and dominance effects in which $\mathbf{G}_{i}$ are additive and dominance relationship matrices is represented by this model. Also, in certain cases, herd or farm effect may be regarded as random in the simple animal model containing breeding value and residual terms.

In this paper, assuming no covariances between the random vectors $\mathbf{u}_{i}$ and $\mathbf{u}_{j}$, we have considered the mixed linear model with multiple components of variance. However, some models frequently used in animal breeding applications may include such covariances. The AI expression of the current type corresponding to a maternal animal model, in which only the covariance between the random vectors of direct and maternal genetic effects is assumed, has been discussed by Ashida and Iwaisaki ${ }^{2}$.

The iterative procedure using equation (22) is categorized as a quasi-Newton type method (e.g., Searle ${ }^{14)}$ ). However, (quasi-) Newton type methods do not always guarantee the estimates within the parameter space. When the use of equation (22) fails to give the globally converged estimates, a globally convergent modification of equation (22) with $A\left(\hat{\theta}_{i}\right)$ 


\section{ASHIDA and IWAISAKI}

replaced by $\boldsymbol{A}\left(\hat{\theta}_{t}\right)+\tau_{t} \mathbf{I}$, where $\tau_{t}$ is a scalar that partially determines the step size and $\mathbf{I}$ is the identity matrix, can be employed ${ }^{9,14)}$ using Marquardt's approach $^{(0)}$. Then, for one round of iteration in the current approach using equation (22) or its modification, the empirical mixed model equations must be solved directly, calculating a generalized inverse of the coefficient matrix. Once the system of the equations are solved, however, no such systems are necessary to be solved in subsequent steps. Instead, to form the current AI matrix, computations of the quadratic and bilinear forms are required. These steps can be handled as a series of calculation of the product of a vector and a matrix, without requiring the computation of the matrices multiplied as is the case with the Fisher scoring method. Furthermore, in the cases of animal breeding applications, the use of sparse matrix tools such as FSPAK ${ }^{12)}$ could make the required calculations efficient. Indeed, the illustrative numerical analysis given in this paper was conducted utilizing the FSPAK subroutines. Also, with use of FSPAK, a data set of carcass records for about two hundred thousand of animals, that approximately includes one hundred thousand of breeding animals in the pedigree file, has been analyzed easily by the current REML approach, using Pentium II (333 MHz) (The Wagyu Cattle Registry Association, personal communication).

A comparison of the computing properties among the current approach and the procedures using other typical algorithms for REML estimation will be reported in another paper.

\section{Acknowledgment}

The authors wish to thank the Wagyu Cattle Registry Association for providing the data.

\section{References}

1) Ashida I, Iwaisaki H. A numerical technique for REML estimation of variance components using average information algorithm and its computing property. Animal Science and Technology, 69:631-636. 1998.

2) Ashida I, Iwaisaki $H$. Average information REML estimation of genetic parameters for market weight of Japanese Black calves. In : Proceedings of the 8th World Conference on Animal Production, Vol. II. 622-623. Seoul National Univ. Seoul. 1998.

3) Dempster AP, Laird NM, Rubin DB. Maximum likelihood from incomplete data via the EM algorithm. Journal of the Royal Statistical Society, Series B 39 : 1-38. 1977.

4) Graser H-U, Smith SP, Tier B. A derivative-free approach for estimating variance components in animal models by restricted maximum likelihood. Journal of Animal Science, 64 : 1362-1370. 1987.

5) Harville DA. Maximum likelihood approaches to variance component estimation and to related problems. Journal of the American Statistical Association, 72 : 320-338. 1977.

6) Henderson $C R$. Sire evaluation and genetic trends. In : Proceedings of the Animal Breeding and Genetics Symposium in Honor of Dr. J. L. Lush. 10-41. American Society of Animal Science and American Dairy Science Association. Champaign. 1973.

7) Henderson CR. Best linear unbiased estimation and prediction under a selection model. Biometrics, 31 : 423-447. 1975.

8) Henderson CR. Applications of Linear Models in Animal Breeding. 17-72. Univ. of Guelph Press. Guelph. 1984.

9) Johnson DL, Thompson R. Restricted maximum likelihood estimation of variance components for univariate animal models using sparse matrix techniques and average information. Journal of Dairy Science, $78:$ :449-456. 1995.

10) Marquardt DW. An algorithm for least-squares estimation of non-linear parameters. Society for Industrial and Applied Mathematics Journal on Applied Mathematics, 11 : 431-441. 1963.

11) Patterson HD, Thompson R. Recovery of inter-block information when block sizes are unequal. Biometrika, 58 : 545-554. 1971.

12) Perez-Enciso M, Misztal I, Elzo ML. FSPAK - An interface for public domain sparse matrix subroutines. Univ. of Georgia. Athens. 1994.

13) Searle SR. Notes on variance components estimation -A detailed account of maximum likelihood and kindred methodology. Technical Report BU-673-M, Biometrics Unit, Cornell Univ. Ithaca. 1979.

14) Searle SR, Casella G, McCulloch CE. Variance Components. 292-297. John Wiley \& Sons. New York. 1992.

15) Smith SP, Graser H-U. Estimating variance components in a class of mixed models by restricted 
An Expression for Average Information Matrix

maximum likelihood. Journal of Dairy Science, 69 : 1156-1165. 1986.

16) Thompson R. Iterative estimation of variance com- ponents for non-orthogonal data. Biometrics, 25 : 767-773. 1969. 